Revista de Matemática: Teoría y Aplicaciones 2007 14(1) : 101-104

CIMPA - UCR - CCSS ISSN: 1409-2433

\title{
A NOTE ON DIRICHLET AND FEJÉR KERNELS
}

\author{
José López-Bonilla* SERgio Vidal Beltrán ${ }^{\dagger}$ \\ JESÚS YALJA MONTIEL ${ }^{\ddagger}$
}

Recibido/Received: 26 Jan 2007 - Aceptado/Accepted: 26 Mar 2007

\begin{abstract}
We exhibit a trigonometric identity wich implies a link between the kernels of Dirichlet and Fejér.
\end{abstract}

Keywords: Fourier Series, Fejér and Dirichlet Kernels; Chebyshev polynomials.

\section{Resumen}

Exhibimos una identidad trigonométrica que implica una relación entre los núcleos de Dirichlet y de Fejér.

Mathematics Subject Classification: 42A20, 42A24, 43A50.

\section{Introduction}

In the original approach to Fourier series [1], it is convenient to consider the following partial sums for the interval $[-\pi, \pi]$ :

$$
f_{n}(y)=\frac{1}{2} a_{0}+a_{1} \cos y+\cdots+a_{n} \cos (n y)+b_{1} \sin y+\ldots b_{n} \sin (n y)
$$

assuming for $a_{r}, b_{r}$ the values:

$$
a_{m}=\frac{1}{\pi} \int_{-\pi}^{\pi} f(t) \cos (m t) d t, \quad b_{m}=\frac{1}{\pi} \int_{-\pi}^{\pi} f(t) \sin (m t) d t
$$

\footnotetext{
* Sección de Estudios de Posgrado e Investigación Escuela Superior de Ingeniería Mecánica y Eléctrica Instituto Politécnico Nacional Edif. Z-4, 3er Piso, Col. Lindavista, C.P. 07738 México, D.F., México. E-Mail: jlopezb@ipn.mx

${ }^{\dagger}$ Misma dirección de J. López. E-Mail: svidalb@ipn.mx

${ }^{\ddagger}$ Escuela Superior de Cómputo, Instituto Politécnico Nacional, Av Bátiz s/n, Col. Nueva Industrial Vallejo, 07738, México D.F., México. E-Mail: yalja@ipn.mx
} 
and investigate what happens if $n$ increases to infinity. From (1.1) and (1.2) we obtain:

$$
f_{n}(y)=\int_{-\pi}^{\pi} f(t) \underset{D}{K_{n}}(t-y) d t
$$

with the Dirichlet kernel [2]-[4]:

$$
\underset{D}{K_{n}}(\theta)=\frac{1}{2 \pi} \frac{\sin \left(n+\frac{1}{2}\right) \theta}{\sin \frac{\theta}{2}}
$$

Then the convergence:

$$
\lim _{n \rightarrow \infty} f_{n}(y)=f(y)
$$

has to be restricted to a definite class of functions $f(y)$ verifying the known Dirichlet conditions [2]-[4].

Fejér [5] invented a new method of summing the Fourier series by which he greatly extended the validity of the series. Using the arithmetic means of the partial sums (1.1), instead of the $f_{n}(y)$ themselves, he could sum series which were divergent. The only condition the function still has to satisfy is the natural restriction that $f(y)$ shall be absolutely integrable. Then, in the Fejér approach we construct the sequence:

$$
\begin{gathered}
g_{1}(y)=f_{0}(y), \quad g_{2}(y)=\frac{1}{2}\left[f_{0}(y)+f_{1}(y)\right], \quad g_{3}(y)=\frac{1}{3}\left[f_{0}(y)+f_{1}(y)+f_{2}(y)\right], \ldots, \\
g_{n}(y)=\frac{1}{n}\left[f_{0}(y)+f_{1}(y)+\cdots+f_{n-1}(y)\right],
\end{gathered}
$$

accepting the expressions (1.1) and (1.2), therefore:

$$
g_{n}(y)=\int_{-\pi}^{\pi} f(t) \underset{F}{K_{n}}(t-y) d t
$$

thus we see that Fejér's results come about by the fact that his method is related with the following Kernel [2]-[4],[5]:

$$
\underset{F}{K_{n}}(\theta)=\frac{1}{2 \pi n} \frac{\sin ^{2}\left(n \frac{\theta}{2}\right)}{\sin ^{2}\left(\frac{\theta}{2}\right)}
$$

then a $f(y)$ absolutely integrable in $[-\pi, \pi]$ guarantess the convergence of $g_{n}(y)$ towards $f(y)$.

In the next section we exhibit a trigonometric identity which shows relationships between the kernels of Dirichlet and Fejér.

\section{$2 \quad$ Links between Fejér and Dirichlet kernels}

It is possible to demonstrate the following identity:

$$
\frac{\sin (2 n+1) \frac{\theta}{2}}{\sin \frac{\theta}{2}}=\frac{1}{\sin ^{2} \frac{\theta}{2}}\left[\sin ^{2}(n+1) \frac{\theta}{2}-\sin ^{2}\left(n \frac{\theta}{2}\right)\right],
$$


which in terms of (1.4) and (1.7) means:

$$
\underset{D}{K_{n}(\theta)}=(n+1) \underset{F}{K_{n+1}}(\theta)-\underset{F}{n K_{n}}(\theta),
$$

that is, the Fejér kernel generates to Dirichlet kernel. For example:

$$
\begin{aligned}
& \underset{D}{K_{0}}=\underset{F}{K_{1}}, \quad \text { because } \quad \underset{F}{K_{0}}=0, \\
& \left.\underset{D}{K_{1}}=\underset{F}{2} \underset{F}{K_{2}}-\underset{F}{K_{1}} \quad \therefore \quad \underset{F}{K_{2}}=\frac{1}{2} \underset{D}{\left(K_{0}\right.}+\underset{D}{K_{1}}\right), \\
& \left.\underset{D}{K_{2}}=\underset{F}{3 K_{3}}-\underset{F}{2 K_{2}} \quad \therefore \quad \underset{F}{K_{3}}=\frac{1}{3} \underset{D}{K_{0}}+\underset{D}{K_{1}}+\underset{D}{K_{2}}\right), \quad \text { etc. }
\end{aligned}
$$

then the inverse relation to (2.2) adopts the form:

$$
\underset{F}{K_{n+1}}(\theta)=\frac{1}{n+1} \sum_{r=0}^{n} \underset{D}{K_{r}(\theta), \quad n=0,1,2, \ldots}
$$

thus the Dirichlet kernel permits to construct the Fejér kernel.

On the other hand, the fourth-kind Chebyshev polynomials are given by $[6],[7]$ :

$$
W_{n}(x)=W_{n}(\cos \theta) \equiv \underset{D}{2 \pi K_{n}(\theta)}=\frac{\sin \left(n+\frac{1}{2}\right) \theta}{\sin \frac{\theta}{2}},
$$

with $\theta \epsilon[0, \pi]$ and $x \epsilon[-1,1]$, therefore:

$$
\begin{gathered}
W_{0}=1, \quad W_{1}=2 x+1, \quad W_{2}=4 x^{2}+2 x-1, \\
W_{3}=8 x^{3}+4 x^{2}-4 x-1, \quad W_{4}=16 x^{4}+8 x^{3}-12 x^{2}-4 x+1, \quad \text { etc. }
\end{gathered}
$$

The relationship (2.3) is important because with it we can introduce new polynomials, in fact:

$$
\begin{aligned}
\tilde{W}_{n}(x) & =\tilde{W}_{n}(\cos \theta) \equiv 2 \pi(n+1) K_{F+1}(\theta)=\frac{\sin ^{2}(n+1) \frac{\theta}{2}}{\sin ^{2} \frac{\theta}{2}} \\
& =\sum_{r=0}^{n} W_{r}(x),
\end{aligned}
$$

that we name "fifth-kind Chebyshev polynomials", which are not explicitly in the literature. For example, (2.5) and (2.7) imply the expressions:

$$
\begin{gathered}
\tilde{W}_{0}=1, \quad \tilde{W}_{1}=2 x+2, \quad \tilde{W}_{2}=4 x^{2}+4 x+1, \\
\tilde{W}_{3}=8 x^{3}+8 x^{2}, \quad \tilde{W}_{4}=16 x^{4}+16 x^{3}-4 x^{2}-4 x+1, \quad \text { etc. }
\end{gathered}
$$


and some of their properties are:

$$
\begin{gathered}
\tilde{W}_{n}(x)=(n+1) \sum_{r=1}^{n+1} \frac{2^{r}}{n+r+1}\left(\begin{array}{c}
n+r+1 \\
2 r
\end{array}\right)(x-1)^{r-1}, \quad \tilde{W}_{n}(1)=(n+1)^{2}, \\
\tilde{W}_{n+1}(x)=2+2 x \tilde{W}_{n}(x)-\tilde{W}_{n-1}(x), \\
\sum_{r=0}^{\infty} \tilde{W}_{r}(x) z^{r}=\frac{1+z}{\left[(1-z)\left(1-2 x z+z^{2}\right)\right]}, \quad|z|<1, \\
(x-1) \tilde{W}_{n}(x)=2^{n} \sum_{r=0}^{n+1}\left(\begin{array}{c}
n+1 \\
r
\end{array}\right)_{2} F_{1}\left(r-n-1,-n-\frac{1}{2} ;-2 n-1 ; 2\right) x^{r}-1 .
\end{gathered}
$$

In a forthcoming work we will study some related topics such as Rodrigues formula, orthonormality, interpolation properties, etc., for this new set of polynomials $\tilde{W}_{n}(x)$.

\section{References}

[1] Roland, N.G. (2000) "Fourier and wavelet representations of functions", Undergrad. J. Math. 6: 1-12.

[2] Lanczos, C. (1966) Discourse on Fourier Series. Oliver \& Boyd, Edinburgh.

[3] Lanczos, C. (1988) Applied Analysis. Dover, New York.

[4] Rodríguez del Río, R.; Zuazua, E. (2003) "Series de Fourier y fenómeno de Gibbs", Cubo Mat. Educ. 5: 185-224.

[5] Fejér, L. (1904) "Untersuchen über Fouriersche Reihen", Math. Annalen 58: 51-69.

[6] Gautschi, W. (1992) "On mean convergence of extended Lagrange interpolation", J. Comp. Appl. Math. 43: 19-35.

[7] Mason, J. C.; Handscomb, D. C. (2002) Chebyshev Polynomials. Chapman \& HallCRC Press, London. 